\title{
A comparison of the available phosphodie- sterase- 5 inhibitors in the treatment of erectile dysfunction: a focus on avanafil
}

This article was published in the following Dove Press journal:

Patient Preference and Adherence

12 August 2015

Number of times this article has been viewed

\section{Jeffery D Evans}

Stephen R Hill

Department of Clinical Sciences, College of Health and Pharmaceutical Sciences, University of Louisiana at Monroe, Monroe, LA, USA
Correspondence: Jeffery D Evans Department of Clinical Sciences, College of Health and Pharmaceutical Sciences, University of Louisiana at Monroe, I725 Claiborne Ave, Shreveport, LA 7II 03 USA

Email jevans@ulm.edu
Background: Numerous pathways can lead to erectile dysfunction (ED) in patients, with some patients having multiple causes. Regardless of the etiology, ED has been successfully treated in many patients with the advent of oral phosphodiesterase-5 inhibitors (PDE5Is). With the release of avanafil, there are currently four PDE5I options available, and choosing between them should be based on patient-specific considerations and preferences.

Objective: To review the treatment of ED with PDE5Is, taking into account the effectiveness, safety, and patient satisfaction of these agents, as well as avanafil's place in therapy.

Methods: A PubMed search was completed to find articles published in English studying patient satisfaction and adherence to ED medication. Additional searches looked specifically for any data regarding the use of avanafil.

Results: ED is effectively treated in most patients with PDE5Is, with the most common side effects from the medications being headache, flushing, and visual disturbances. Patients have identified many different factors, such as efficacy, side effects, duration of action, and daily use, in determining overall satisfaction and the right medication for them. While avanafil does not have any patient satisfaction trials to date, it has been proven to be a safe and effective treatment for ED with possibly the fastest onset of action and fewer visual disturbances than its competitors.

Conclusion: Avanafil along with the other PDE5Is has shown to be a safe and effective oral treatment for ED, with avanafil's possible place in therapy for patients who want an on-demand option or as an alternative in patients who experience visual disturbances with the other agents.

Keywords: erectile dysfunction, avanafil, PDE51, sildenafil, vardenafil

\section{Introduction}

Erectile dysfunction (ED) continues to be a cause of outpatient office visits. In the USA, approximately $18.4 \%$ of males over the age of 20 years report having ED according to 2001-2002 National Health and Nutrition Examination survey data. ${ }^{1}$ Rates of patients reporting "sometimes able" to have normal erectile function and "never able" to have normal erectile function were $33.7 \%$ and $36.5 \%$, respectively, in patients older than 70 years. Two later surveys showed similar findings, one conducted in the USA and the other in Europe. The USA study ${ }^{2}$ published in 2007 reported $>40 \%$ of men above the age of 64 years had experienced difficulty in achieving or maintaining an erection. In the European study ${ }^{3}$ published in 2010 , greater than $30 \%$ of surveyed men who were 60 years or older reported moderate or severe ED. Also, many cardiovascular risk factors such as hypertension, diabetes mellitus, hyperlipidemia, physical inactivity, and smoking increase the frequency of ED. ${ }^{1}$ 


\section{Background on causes of ED}

ED can result from the interaction of multiple physiologic systems, and many patients will have more than one cause of ED. The different causes include neurogenic, endocrinologic, vasculogenic, medication- or substance-induced, poor general or cardiovascular health, and local penile issues such as penile fracture. ${ }^{4}$ Also psychogenic causes such as trauma from poor prior sexual experiences, relationship problems, and low self-esteem (especially when associated with sexual function) may induce ED. When a patient is being evaluated for ED, it is important to consider all of the potential problems that may contribute to the dysfunction and develop a treatment plan to address each of the problems with the patient and potentially the partner. It should also be noted that ED appears to be an early predictor of cardiovascular disease and patients with ED may warrant additional evaluation even if other symptoms are absent at the initial presentation.

\section{Treatments of ED}

Over time, several treatments have been developed for patients with ED, with yohimbine being one of the oldest. It has been used to treat ED with limited success along with other agents such as phentolamine. Alprostadil, a prostaglandin E-1 (PGE1) analog, is very effective for the treatment of ED with success rates as high as $70 \%$; however, since it must be administered either intracavernosally or intraurethrally, it is not as appealing as taking a medication by mouth. ${ }^{4} \mathrm{With}$ the approval of the phosphodiesterase-5 inhibitors (PDE5Is) that could be taken orally, the treatment of ED became a more attractive therapy with fewer side effects and similar efficacy. However, PDE5I therapy does have limitations especially with patients who may need organic nitrates for the treatment of angina.

Reasons for patients to discontinue PGE1 analogs have been studied and can be tied to the administration route. A study of Korean men who were either taking a PGE1 ana$\log$ or had been taking one describes the causes of discontinuation and why some patients continue the medication. This trial ${ }^{5}$ involved 294 men who were prescribed a PGE1 analog to be injected intracavernosally. The trial included 59 patients who had continued the medication for an average of 56 months and 235 patients who had stopped therapy with a PGE1 analog after an average of 18 months. Demographically, the patients were essentially the same age and had many of the same comorbid disease states. Differences arose first in the reported side effects experienced by the two groups. In patients who discontinued the medication, pain with administration was reported by $45.9 \%$ of the patients while prolonged erection was reported by $23 \%$ of patients. In the group that continued the medication, only $24 \%$ of patients reported pain with administration but $44 \%$ reported prolonged erection. Other adverse events including penile curvature and palpable plaque were nearly equal between the two groups. If a patient stopped the medication due to side effects, pain was the most frequently reported side effect occurring $47.9 \%$ of the time while prolonged erection was $21.7 \%$ of the time. However, the most frequently reported reason for stopping the PGE1 analog was a lack of efficacy as reported by $43.1 \%$ of patients who discontinued the medication. Patients who stopped the PGE1 analog reported switching to PDE5Is $63.1 \%$ of the time or a vacuum device $21.1 \%$ of the time. The patients in this trial were using PGE1 analogs at the time sildenafil was introduced, thus partially explaining why the patients had not tried a PDE5I prior to trying intracavernosal PGE1 analogs.

\section{Description of a "perfect" ED medication}

A medication used to treat ED should have predictable efficacy and time of onset, should be well tolerated and easy to administer, and should be associated with minimal side effects. For patients taking an ED medication, they should have the option to choose a medication that works immediately and then wears off, or take a medication that may be slightly delayed in its effects but remains effective for hours. In recent years, the definition has changed to include medications that could be taken every day instead of only when needed. The medication must be of good cost-value for the patient, and the prescriber must be familiar with the medication's limitations.

\section{PDE5 inhibitors available for patient use \\ Sildenafil}

Sildenafil was the original PDE5I to be released and has been extensively researched. It has a quick onset of action of 30 minutes after the initial dose, although patients are instructed to wait at least 1 hour prior to attempting intercourse to receive the most benefit from the medication. It has been shown to have a duration of action of 4-6 hours and a maximum duration of 12 hours. ${ }^{6}$ Visual disturbances have been reported more commonly with sildenafil over the other PDE5Is, most likely due to its affinity for PDE6 inhibition. Although the patent for Viagra (sildenafil), originally released in 1998, has been extended to 2020 in the USA after 
winning a court case against a generic manufacturer, Pfizer has entered into an agreement to allow a generic company to market sildenafil in $2017 .^{7}$

\section{Tadalafil}

Tadalafil was released in 2003 making it the third PDE5I on the market for the treatment of ED. It has an onset of 20 minutes and should be taken 30 minutes prior to intercourse. Tadalafil has the longest duration of action in the class (24-36 hours) and a reported maximum duration of 72 hours. Tadalafil is approved for once daily use for the treatment of ED and benign prostate hyperplasia. ${ }^{4}$ Trials have shown significant increases in the rates of erectogenic response as soon as 15 minutes after administration of tadalafil $20 \mathrm{mg}$ and approximately 30 minutes after administration of tadalafil $10 \mathrm{mg}$. Fifty-two percent of men in the study were able to have successful intercourse within 30 minutes of taking tadalafil $20 \mathrm{mg} .{ }^{9}$ Cialis (tadalafil) is expected to become generically available in $2017 .{ }^{8}$

\section{Vardenafil}

Vardenafil was approved in 2003 for the treatment of ED. It has the shortest reported onset of action (10 minutes), but it is recommended to be used within 30-60 minutes of planned intercourse. The duration of action is between 5 and 7 hours and has a maximum duration of 12 hours. ${ }^{6}$ Vardenafil may lengthen the QTc interval and must be used with caution or avoided in patients with prolonged QTc or those currently taking Class 1 or 3 antiarrhythmics. Levitra (vardenafil) patents begin to expire in $2018 .^{8}$

\section{Avanafil}

Avanafil (Stendra) is the newest of the PDE5Is on the market. It was USFDA approved in 2012 and became the fourth PDE5I on the market. Avanafil was generally well-tolerated during clinical trials with the most common treatment-related adverse events being headache and flushing, although fewer than $14 \%$ of patients reported these side effects. ${ }^{10} \mathrm{With}$ only 3 years of usage, there are no identifiable patient satisfaction studies in print.

Avanafil reaches a maximum concentration $\left(T_{\max }\right)$ within 30-45 minutes of oral administration and has an adjusted $T_{\max }$ of up to 1.25 hours if a high-fat meal is ingested with the medication. ${ }^{10}$ The medication undergoes metabolism through cytochrome P450 3A4 enzyme and produces both inactive and active metabolites. The terminal half-life of avanafil is approximately 5 hours with the majority of the medication excreted in the feces. The half-life of avanafil is slightly longer than sildenafil and vardenafil but only about a third of tadalafil.
At the time of its release in the European Union and US, avanafil had been evaluated in approximately 1,300 patients. ${ }^{11}$ The medication was termed as a second-generation PDE5I with a higher selectivity for PDE5 versus other agents that had additional attraction to other subtypes of PDE receptors. With increased selectivity, it was hoped that the medication would be more effective and have fewer visual side effects tied to PDE6. During the preapproval trials, one case of vision changes associated with other PDE5Is occurred, which is a lower rate than the other PDE5Is currently on the market. No cases of EKG changes, such as QTc prolongation occurred during the trials.

Avanafil's effectiveness has been studied in 12-week trials showing that it was effective for the short-term, on-demand treatment of ED. Patients were randomized to receive either 50,100, or $200 \mathrm{mg}$ doses 30 minutes prior to sexual intercourse. Patients were expected to have intercourse at least four times per month. For research purposes, patients completed a survey once in every 4 weeks to determine the effectiveness of the medication. Patients taking any dosage of the medication had a statistically significant increase in both successful vaginal penetration and successful intercourse. The study also evaluated the time from dosage to intercourse, which found that patients taking the medication were more successful than patients taking a placebo at all times. Approximately $70 \%$ of patients reported that they were able to have intercourse within 15 minutes of taking a dose of avanafil. The medication was still effective for $>6$ hours after taking the medication as the rate of successful sexual intercourse occurred between 59\% and $83 \%$ depending on the dose. Survey research showed that patients taking the $200 \mathrm{mg}$ dose had higher rates of orgasmic function, sexual desire, intercourse satisfaction, and overall satisfaction. ${ }^{12}$

Avanafil has been evaluated in a long-term trial studying its effects in patients with varying degrees of ED and the side effects associated with it. All patients in the trial began with the $100 \mathrm{mg}$ dose taken as needed 30 minutes prior to the anticipated sexual intercourse. The trial had 711 patients who were on average over the age of 55 years and had reported having ED for $>5$ years. More than $75 \%$ of patients in the trial requested to increase their dose to $200 \mathrm{mg}$ per encounter due to a lack of efficacy of the $100 \mathrm{mg}$ dose. However, showing that either avanafil dose is well-tolerated, only three patients requested that the dosage of the medication should be reduced to $50 \mathrm{mg}$ in response to intolerable side effects. The most common adverse effects reported in the trial were headache and flushing. ${ }^{13}$ 
Avanafil has also been evaluated for very quick use in patients with mild-to-severe ED. Patients were randomized to either placebo or avanafil $100 \mathrm{mg}$ or $200 \mathrm{mg}$. Patients were instructed to take the medication with or without food and begin sexual intercourse within 15 minutes of taking the medication. Significant differences in patients with successful intercourse attempts occurred at 10 minutes for the $200 \mathrm{mg}$ dose and 12 minutes for the $100 \mathrm{mg}$ dose compared to placebo. But it is important to note that the rates for successful intercourse were only $26 \%$ for the $100 \mathrm{mg}$ dose and $29 \%$ for the $200 \mathrm{mg}$ dose. Both of these values are significantly below the expected success rates as seen in the earlier trials. In this trial, the longer the patients waited between taking the medication and beginning sexual intercourse, the higher the chances were for a successful sexual encounter with both doses of the medication. The most common treatment-related side effects in this trial were headache (3.7\%) and upper respiratory tract infections (3.1\%). Rates of side effects were higher in the $200 \mathrm{mg}$ dose group compared to the $100 \mathrm{mg}$ dose group. ${ }^{14}$

A meta-analysis was completed for each of the approved PDE5Is comparing effectiveness and safety. ${ }^{15}$ The trial only included two avanafil studies out of the 118 trials evaluated; thus, data with avanafil in this analysis may be inaccurate. However, whereas effectiveness seems comparable to the other PDE5Is, the rates of side effects were the highest in the avanafil group. The risk ratio $(95 \% \mathrm{CI})$ compared to placebo for any adverse event for avanafil was 2.43 (1.35-4.30) compared to sildenafil 1.99 (1.47-2.61), tadalafil 2.09 (1.41-3.07), and vardenafil 2.03 (1.48-2.66).

\section{Evaluation of patient satisfaction with erectile dysfunction treatments}

Patient preference will ultimately determine if a patient continues the medication. In patients with ED, $60 \%-70 \%$ of the population will see a positive outcome from the use of a PDE5I. Yet, $30 \%-40 \%$ of these initial responders state that they are unhappy with on-demand treatment of ED. ${ }^{16}$ Half of the initial responders will stop the medications within the first 2-3 years. Regardless of which of the PDE5Is are chosen, significant improvements can be found to both the patient's and partner's sexual lives. ${ }^{17}$ Both Self-Esteem and Relationship and International Index of Erectile Function scores can improve for patients who start PDE5I for the first time. The Self-Esteem and Relationship subdomains for self-esteem, overall relationship, and sexual relationship improve significantly with initial therapy for the patient. Sexual drive of the partner was not increased; however, sexual and general life satisfaction did improve. The partners of patients who had the largest increases in self-esteem showed the largest increases in sexual and general satisfaction. Approximately $67 \%$ of general practitioners who prescribed PDE5I had no worries about prescribing PDE5Is and about half of surveyed practitioners could differentiate between the various PDE5Is.

A large, multicenter study evaluated patient preference and effectiveness over a 6-month period. ${ }^{18}$ The study was completed prior to avanafil being introduced, and the drug is not discussed in this trial. In this large (8,047 patients) observational trial, the majority of patients were taking a PDE5I at the start of the trial. The most common agent reported was tadalafil, with approximately $66 \%$ of patients using this PDE5I. During the 6 months, about half of the patients continued taking the same medication. The patients had a mean age of 56 years and most had moderate ED at the start of the trial. Most patients had mixed etiology, both organic and psychogenic ED for more than 1 year. The study found that all patients who chose a PDE5I had improved patient satisfaction and sexual performance compared to those not having treatment at baseline. Patients receiving tadalafil reported the highest level of patient satisfaction and therapeutic effectiveness, with vardenafil and sildenafil having similar effectiveness. The researchers also surveyed the patients to find out what the longest period of time between taking a PDE5I and having a successful intercourse was during the trial period. Both patient groups taking vardenafil or sildenafil reported that the longest time that had elapsed for at least $90 \%$ of the groups was approximately 12 hours or less. In the tadalafil group, $90 \%$ of patients reported 48 hours or less between taking a dose of the medication and a successful sexual intercourse. This additional aspect of the medication may have played a role in the higher levels of patient satisfaction with the tadalafil compared to the other agents.

Another trial evaluated men in Central and South America using various PDE5Is. The trial ${ }^{19}$ was a subset of data that focused on men in Brazil during a 6-month observational period researching which PDE5Is patients were more likely to be adherent with. The study allowed any available PDE5I to be used. The most common one chosen at baseline was sildenafil, with tadalafil being second and vardenafil being the least frequent choice. The study found that patients were more likely to be persistent and adherent to tadalafil at each of the time markers observed (1, 3, and 6 months). Seventyfive percent of patients were adherent and persistent with tadalafil compared to values between 60 and 70 for sildenafil and vardenafil. These differences were not found to be 
statistically different, but the study only had 104 patients enrolled. The study did find that employment status had a significant impact on adherence, possibly indicating that cost of the medications may have mattered.

A survey administered to Japanese men evaluated several aspects of ED's impact on sexual performance and anxiety. The survey was completed by 546 men who were 30-70 years old with the majority of the patients being between 40 and 60 years of age. Approximately $40 \%$ of the patients who completed the survey were taking sildenafil mostly during the time of the survey. Approximately $30 \%$ of patients were taking either vardenafil or tadalafil. No dosages were recorded for any of the patients. There were significant differences between sildenafil and either vardenafil or tadalafil in the subdomains of self-confidence and spontaneity. In both of these subdomains, both vardenafil and tadalafil patients were more likely to provide a higher rating than the patients who were taking sildenafil. Neither vardenafil nor tadalafil were found to be significantly better than the other. Tadalafil was reported to be inferior to vardenafil or sildenafil in the subdomain of time concern, and neither vardenafil nor sildenafil was superior to each other in this subdomain. One weakness of the trial is that the sildenafil $100 \mathrm{mg}$ dose is not approved in Japan, but the maximum doses of other medications are approved. ${ }^{20}$ Determining the impact of dosing type in initial adherence may also point to what patients want from a PDE5I. A study comparing once daily tadalafil, as-needed tadalafil, and on-demand sildenafil included 520 patients divided between the three groups at baseline. Patients had not previously been treated with any PDE5I. Patients were allowed to switch medications and to titrate doses as needed. Patients were more likely to request a dose increase if they started in the sildenafil $50 \mathrm{mg}$ group, and few patients requested a dose reduction. Sildenafil patients were the quickest to discontinue therapy with a median time to discontinuation of 67 days. Tadalafil on demand had the longest median time to discontinuation ( $>168$ days), as the trial did not continue long enough for $50 \%$ of patients stopped the medication. Tadalafil once daily had a median discontinuation time of 130 days. Patients reported discontinuing the medication most frequently due to lack of efficacy, which was reported equally between each of the groups. However, the duration of erection, which was the second most reported reason for discontinuation, was more common with sildenafil (9.2\%) and tadalafil once daily $(4.3 \%)$. Ten percent of patients in the tadalafil once-daily group stopped taking the medication because they did not want to take the medication daily, and $10 \%$ of patients in the sildenafil group did not want to take a medication as needed. This study demonstrates the need to tailor a regimen to meet the needs of the patient. ${ }^{21}$

Although not considered in the trials described earlier, some trials have looked at partner satisfaction with PDE5I therapy. The Sexual Encounter Profile questionnaire and the modified Sexual Life Quality Questionnaire (mSLQQ-QOL) were administered to the partners of patients using either vardenafil with flexible dosing or placebo. Partners of study participants were surveyed at baseline and during the final week of the trial. At baseline using the MSLQQ-QOL, the partner's values were below the participant's values. At week 12 in the vardenafil group, both partner and participant's scores had significantly increased and were essentially the same. Based upon the MSLQQ-QOL results from the vardenafil treatment group, both partner and participant felt that their sex lives were comparable to their sex lives prior to the onset of ED. ${ }^{22}$

\section{Avanafil's place in therapy}

As the latest medication in the class, avanafil needs to show a benefit over other PDE5Is to gain market share. Avanafil is positioning itself to be the quickest acting of the PDE5I, thus you can take it at the moment that you need it. However, while the labeling for the other PDE5Is may not indicate it, they also may be acting quicker than what was originally thought. Furthermore, vardenafil may actually work quicker than avanafil and provide efficacy for just as long. Tadalafil, which provides a once daily option, works approximately 10 minutes slower than avanafil, and then stays in the body longer providing the patient a wider window of effectiveness than avanafil, which may be viewed as a strength or weakness. Finally, compared to sildenafil, avanafil is a better option with a quicker onset and similar duration. Avanafil was studied and is approved to be used without regard to food or alcohol intake, whereas some other PDE5Is are not. This benefit may increase patient satisfaction with avanafil, but will probably be limited in the number of patients seeing this as a benefit.

Cost and access is another concern with avanafil that may hamper patient's satisfaction. The agents in this class of medication are all priced similarly. Two of the PDE5Is that were released prior to avanafil are going to have generic alternatives starting in 2017. By the middle of 2018, avanafil may be the only branded PDE5I on the market unless a successful patent defense is made by the patent holder to extend the amount of time for one of the other medications. Thus, with other agents costing less than avanafil, patient satisfaction may decrease due to the cost difference. 


\section{Conclusion}

PDE5Is have become the mainstay and first-line treatment for ED in men. The classes' overall effectiveness, ease of use by mouth, quick onset of action, and tolerable side effect profile have helped them become one of the most popular medications prescribed over the last 15 years. Patients and their partners have identified an improved sex life and higher patient satisfaction scores when taking a PDE5I. Avanafil is the fourth PDE5I released to the market and has the potential advantages of taking it closer to the time of sexual activity and less visual disturbances than its in-class competitors. Avanafil is currently priced similarly to the other PDEIs, but as the older agents transition to generic alternatives the price difference will most likely become more pronounced and favor the generic options.

\section{Disclosure}

The authors report no conflicts of interest in this work.

\section{References}

1. Selvin E, Burnett AL, Platz EA. Prevalence and risk factors for erectile dysfunction in the US. Am J Med. 2007;120:151-157.

2. Lindau ST, Schumm LP, Laumann EO, Levinson W, O'Muircheartaigh CA, Waite LJ. A study of sexuality and health among older adults in the United States. N Engl J Med. 2007;357:762-774.

3. Corona G, Lee DM, Forti G, et al; EMAS Study Group. Age-related changes in general and sexual health in middle-aged and older men: results from the European male ageing study. J Sex Med. 2010;7: $1362-1380$.

4. Shamloul R, Ghanem H. Erectile dysfunction. Lancet. 2013;381: 153-165.

5. Sung HH, Ahn JS, Kim JJ, Choo SH, Han DH, Lee SW. The role of intracavernosal injection therapy and the reasons of withdrawal from therapy in patients with erectile dysfunction in the era of PDE5 inhibitors. Andrology. 2014;2:45-50.

6. Raheem AA, Kell P. Patient preference and satisfaction in erectile dysfunction therapy: a comparison of the three phosphodiesterase-5 inhibitors sildenafil, vardenafil and tadalafil. Patient Prefer Adherence. 2009;3:99-104.

7. Amendola E. Viagra to go generic in 2017 according to Pfizer agreement; 2013. Available from: cbsnews com [serial online]. Available from: CBS News. Accessed February 14, 2015.

8. Phillips DJ. Pfizer's Expiring Viagra Patent Adversely Affects Other Drugmakers Too. Available from: Forbes.com. December 20, 2013, February 13, 2015. [Ref Type: Online Source].
9. Rosen RC,Padma-Nathan H, Shabsigh R, Saikali K, Watkins V, Pullman W. Determining the earliest time within 30 minutes to erectogenic effect after tadalafil 10 and $20 \mathrm{mg}$ : a multicenter, randomized, double-blind, placebo-controlled, at-home study. J Sex Med. 2004;1:193-200.

10. Kyle JA, Brown DA, Hill JK. Avanafil for erectile dysfunction. Ann Pharmacother. 2013;47:1312-1320.

11. Burke RM, Evans JD. Avanafil for treatment of erectile dysfunction: review of its potential. Vasc Health Risk Manag. 2012;8:517-523.

12. Goldstein I, McCullough AR, Jones LA, et al. A randomized, doubleblind, placebo-controlled evaluation of the safety and efficacy of avanafil in subjects with erectile dysfunction. $J$ Sex Med. 2012;9:1122-1133.

13. Belkoff LH, McCullough A, Goldstein I, et al. An open-label, longterm evaluation of the safety, efficacy and tolerability of avanafil in male patients with mild to severe erectile dysfunction. Int J Clin Pract. 2013;67:333-341.

14. Hellstrom WJ, Kaminetsky J, Belkoff LH, et al. Efficacy of avanafil fifteen minutes after dosing in men with erectile dysfunction: a randomized, double-blind, placebo-controlled study. J Urol. 2015;194(2):485-492.

15. Yuan J, Zhang R, Yang Z, et al. Comparative effectiveness and safety of oral phosphodiesterase type 5 inhibitors for erectile dysfunction: a systematic review and network meta-analysis. Eur Urol. 2013; 63:902-912.

16. Smith WB, McCaslin IR, Gokce A, Mandava SH, Trost L, Hellstrom WJ. PDE5 inhibitors: considerations for preference and long-term adherence. Int J Clin Pract. 2013;67:768-780.

17. Costa P, Grandmottet G, Mai HD, Droupy S. Impact of a first treatment with phosphodiesterase inhibitors on men and partners' quality of sexual life: results of a prospective study in primary care. J Sex Med. 2013;10:1850-1860.

18. Martin-Morales A, Haro JM, Beardsworth A, Bertsch J, Kontodimas S. Therapeutic effectiveness and patient satisfaction after 6 months of treatment with tadalafil, sildenafil, and vardenafil: results from the erectile dysfunction observational study (EDOS). Eur Urol. 2007;51: 541-550.

19. Cairoli C, Reyes LA, Henneges C, Sorsaburu S. PDE5 inhibitor treatment persistence and adherence in Brazilian men: post-hoc analyses from a 6-month, prospective, observational study. Int Braz J Urol. 2014;40: 390-399.

20. Tsujimura A, Kiuchi H, Soda T, et al. Sexual life of Japanese patients with erectile dysfunction taking phosphodiesterase type 5 inhibitors: an Internet survey using the Psychological and Interpersonal Relationship Scales-Short Form questionnaire. Int J Urol. 2014;21:821-825.

21. Buvat J, Büttner H, Hatzimouratidis K, et al. Adherence to initial PDE-5 inhibitor treatment: randomized open-label study comparing tadalafil once a day, tadalafil on demand, and sildenafil on demand in patients with erectile dysfunction. J Sex Med. 2013;10:1592-1602.

22. Martín-Morales A, Graziottin A, Jaoudé GB, et al. Improvement in sexual quality of life of the female partner following vardenafil treatment of men with erectile dysfunction: a randomized, double-blind, Placebo-controlled study. J Sex Med. 2011;8:2831-2840.
Patient Preference and Adherence

\section{Publish your work in this journal}

Patient Preference and Adherence is an international, peer-reviewed, open access journal that focuses on the growing importance of patient preference and adherence throughout the therapeutic continuum. Patient satisfaction, acceptability, quality of life, compliance, persistence and their role in developing new therapeutic modalities and compounds to optimize

\section{Dovepress}

clinical outcomes for existing disease states are major areas of interest for the journal. This journal has been accepted for indexing on PubMed Central. The manuscript management system is completely online and includes a very quick and fair peer-review system, which is all easy to use. Visit http://www. dovepress.com/testimonials.php to read real quotes from published authors. 